\title{
Aneurysm location and clipping versus coiling for development of secondary normal-pressure hydrocephalus after aneurysmal subarachnoid hemorrhage: Japanese Stroke DataBank
}

\author{
Shigeki Yamada, MD, PhD, ${ }^{1}$ Masatsune Ishikawa, MD, PhD, ${ }^{1}$ Kazuo Yamamoto, MD, ${ }^{1}$ \\ Tadashi Ino, MD, PhD, ${ }^{2}$ Toru Kimura, MD, PhD, ${ }^{3}$ Shotai Kobayashi, MD, PhD, ${ }^{4}$ and \\ *Japan Standard Stroke Registry Study Group
}

\begin{abstract}
1Department of Neurosurgery, Stroke Center and Normal Pressure Hydrocephalus Center, and 'Department of Neurology, Rakuwakai Otowa Hospital; ${ }^{3}$ Department of Neurology, Rakuwakai Misasagi Hospital, Kyoto; and ${ }^{4}$ Shimane University, Shimane, Japan
\end{abstract}

\begin{abstract}
OBJECT The present study aimed to investigate aneurysm locations and treatments for ruptured cerebral aneurysms associated with secondary normal-pressure hydrocephalus (sNPH) after subarachnoid hemorrhage (SAH) by using comprehensive data from the Japanese Stroke DataBank.

METHODS Among 101,165 patients with acute stroke registered between 2000 and 2013, 4693 patients (1482 men, 3211 women) were registered as having had an SAH caused by a ruptured saccular aneurysm. Of them, 1448 patients (438 men and 1010 women; mean age $61.9 \pm 13.4$ years) who were confirmed to have or not have coexisting acute hydrocephalus and sNPH were included for statistical analyses. Locations of the ruptured aneurysms were subcategorized into 1 of the following 4 groups: middle cerebral artery $(\mathrm{MCA} ; n=354)$, anterior communicating artery and anterior cerebral artery (ACA; $n=496)$, internal carotid artery (ICA; $n=402)$, and posterior circulation $(n=130)$. Locations of 66 of the ruptured aneurysms were unknown/unrecorded. Treatments included craniotomy and clipping alone in 1073 patients, endovascular coil embolization alone in 285 patients, and a combination of coiling and clipping in 17 patients. The age-adjusted and multivariate odds ratios from logistic regression analyses were calculated after stratification using the Fisher CT scale to investigate the effects of the hematoma volume of $\mathrm{SAH}$.
\end{abstract}

RESULTS Acute hydrocephalus was confirmed in 593 patients, and 521 patients developed sNPH. Patients with a ruptured ACA aneurysm had twice the risk for sNPH over those with a ruptured MCA aneurysm. Those with an ACA aneurysm with Fisher Grade 3 SAH had a 9-fold-higher risk for sNPH than those with an MCA aneurysm with Fisher Grade 1 or $2 \mathrm{SAH}$. Patients with a ruptured posterior circulation aneurysm did not have any significant risk for sNPH. Clipping of the ruptured aneurysm resulted in twice the risk for sNPH over coil embolization alone.

CONCLUSIONS Patients with low-grade SAH caused by a ruptured MCA aneurysm had a low risk for the development of sNPH. In contrast, patients with high-grade SAH caused by a ruptured ACA aneurysm had a higher risk for sNPH. Endovascular coiling might confer a lower risk of developing sNPH than microsurgical clipping.

http://thejns.org/doi/abs/10.3171/2015.1.JNS142761

KEY WORDS secondary NPH; subarachnoid hemorrhage; acute hydrocephalus; endovascular coil embolization; vascular disorders

\footnotetext{
ABBREVIATIONS ACA = anterior cerebral artery; ICA = internal carotid artery; $M C A=$ middle cerebral artery; NPH = normal-pressure hydrocephalus; SAH = subarachnoid hemorrhage; sNPH = secondary NPH; WFNS = World Federation of Neurological Societies.

SUBMITTED December 5, 2014. ACCEPTED January 21, 2015.

INCLUDE WHEN CITING Published online July 31, 2015; DOI: 10.3171/2015.1.JNS142761.

DISCLOSURE Dr. Ishikawa has received honoraria from Medtronic Japan Co., Ltd. (Japan). Dr. Yamada declares no disclosures and no conflicts of interest. Drs. Yamamoto, Ino, Kimura, and Kobayashi report no conflict of interest concerning the materials or methods used in this study or the findings specified in this paper. All authors have made substantial contributions to the intellectual content of the paper, have approved the final manuscript, and agree with submission to this journal. Dr. Yamada is the corresponding author for this study and the principal investigator. He takes responsibility for data management, accuracy of statistical analysis, conduct of the research, and drafting of the manuscript. The following grants funded this study: "21st Century Type Promoting Development of Clinical Research Fund" from the Japanese of Health, Labor and Welfare during 1999 to 2001.

* See Appendix for listing of institutions participating in the Japan Standard Stroke Registry Study Group.
} 
$\mathrm{T}$ HE natural history of secondary normal-pressure hydrocephalus (sNPH) occurring 1 or 2 months after subarachnoid hemorrhage (SAH) is becoming clear. The prevalence of sNPH has been reported to be $8.9 \%-48 \%$ in patients with SAH. ${ }^{1-7,11,12,16,18,22,23}$ Acute hydrocephalus coexisting with $\mathrm{SAH}$ is known to be one of the most important predictors for sNPH. ${ }^{3-6,11,16}$ Previous studies have shown that severe symptoms at SAH onset and a large amount of subarachnoid blood seen on admission CT images are associated with the development of $\mathrm{sNPH}{ }^{3-11,12,16,18,23}$ A posterior circulation location of the ruptured aneurysm and endovascular coil embolization have been reported to be significantly associated with sNPH. 1,4-7,12,15,16,22 However, this relationship is controversial, because SAH caused by a posterior circulation aneurysm frequently occurs with severe initial symptoms and acute hydrocephalus, compared to SAH caused by a ruptured anterior circulation aneurysm, which is known to occur with mild symptoms and/or no acute hydrocephalus. ${ }^{14}$ In addition, most ruptured posterior circulation aneurysms have been treated with endovascular coil embolization., ${ }^{3,5}$ This selection bias in previous hospital-based studies might have influenced the risk for development of sNPH after coil embolization among patients with a ruptured posterior circulation aneurysm. In the present study, by taking an advantage of a large nationwide registration study, we investigated the risk of sNPH associated with aneurysm location and treatment (clipping or coil embolization) for the ruptured cerebral aneurysm.

\section{Methods \\ Population}

Between 2000 and 2013, the Japanese Standard Stroke Registry Study accumulated data on 101,165 patients with acute stroke treated in 163 institutions across Japan. ${ }^{10,20}$ This nationwide stroke database was established with support from the Japanese Ministry of Health, Labor and Welfare to provide evidence for the standardization of Japanese stroke management. The present study was approved by the ethics committee of the Shimane Medical University. Details of data collection and management have been published elsewhere. ${ }^{10,20}$ A total of 5344 patients $(5.3 \%$ of the registered population; 1772 men and 3572 women; mean age $62.5 \pm 14.4$ years) were registered as having had an SAH. Among them, 4693 patients were diagnosed with a ruptured saccular aneurysm, 196 had a dissecting aneurysm, 27 had an infectious or traumatic pseudo-aneurysm, and 428 had an aneurysm for which the origin was unknown or not recorded. In this study, the patients confirmed to have had a ruptured cerebral saccular aneurysm were included because of the different pathological characteristics of dissecting and pseudoaneurysms. In addition, to clarify the relationship between acute hydrocephalus and sNPH, this study included the patients who were confirmed to have or not have coexisting acute hydrocephalus and sNPH, as diagnosed by the attending neurosurgeons at the registered hospitals. We also gathered data on patients diagnosed with acute hydrocephalus who experienced neurological deterioration and underwent immediate CSF diversion by placement of an external ventricular or lumbar drain on admission. The records of patients with
sNPH after SAH were also reviewed to note their symptoms and whether they underwent ventriculoperitoneal or lumboperitoneal shunt placement for permanent CSF diversion. Finally, 1448 patients (438 men and 1010 women; mean age $61.9 \pm 13.4$ years) were included for statistical analyses. The database recorded age, sex, medical history, medication status, family history of stroke, lifestyle factors, neurological severity grading at SAH onset (NIH Stroke Scale, World Federation of Neurological Societies [WFNS] scale, and Hunt and Kosnik scale), Fisher CT scale grade (from the admission CT scan), and treatment for the ruptured cerebral aneurysm (neurosurgical clipping and/or endovascular coil embolization). Locations of the ruptured cerebral aneurysms were categorized as follows: middle cerebral artery (MCA; $\mathrm{n}=354$ ), anterior communicating artery $(n=416)$, anterior cerebral artery (ACA; $n$ $=80$ ), internal carotid-posterior communicating artery (n $=395)$, other internal carotid artery (ICA; $n=7)$, posterior cerebral artery $(n=14)$, basilar tip $(n=48)$, basilar-superior cerebellar artery $(n=21)$, vertebral artery-posterior inferior cerebellar artery $(\mathrm{n}=47)$, and unknown or unrecorded $(n=66)$. The aneurysm locations were subcategorized into 1 of the following 4 groups: MCA, ACA (which included the ACA and the anterior communicating artery), ICA (which included the ICA-posterior communicating artery and other ICAs), and posterior circulation (which included the posterior cerebral artery, basilar tip, basilarsuperior cerebellar artery, and vertebral artery-posterior inferior cerebellar artery).

\section{Statistical Analysis}

Odds ratios and $95 \%$ CIs for the development of sNPH were calculated. Using logistic regression analyses, we investigated the association between SNPH and the following variables: age ( $<60$ or $\geq 60$ years), sex, acute hydrocephalus, craniotomy and microsurgical clipping or endovascular coil embolization, hypertension, diabetes, dyslipidemia, smoking habit, alcohol consumption, WFNS scale grade, Hunt and Kosnik scale grade, and Fisher CT scale grade. All analyses were adjusted by the continuous variable of age at registration. To assess the effects of modification and interaction, we conducted multivariate analyses after adjusting for age, sex, hypertension, smoking habit, acute hydrocephalus, and Fisher CT scale grade. In addition, to investigate the effects of acute hydrocephalus and hematoma volume, age-adjusted and multivariate odds ratios were analyzed after stratification according to acute hydrocephalus (versus no acute hydrocephalus) and Fisher CT scale grade. All missing variables were treated as deficit data that did not change the other variables. Statistical significance was assumed at a Fisher exact test probability value (p) of $<0.05$. Statistical analyses were performed using R software (version 3.1.2, R Foundation for Statistical Computing; http://www.R-project.org).

\section{Results}

The clinical characteristics of the 1448 patients diagnosed with SAH caused by a ruptured cerebral saccular aneurysm are summarized in Table 1 . Of them, 521 were registered as having had $\mathrm{SNPH}$ after aneurysmal SAH, and $444(85 \%)$ of these patients underwent shunt surgery. 
TABLE 1. Clinical characteristics in each location of ruptured cerebral aneurysm on admission*

\begin{tabular}{|c|c|c|c|c|c|c|}
\hline \multirow[b]{2}{*}{ Characteristic } & \multicolumn{6}{|c|}{ Aneurysm Location } \\
\hline & All & MCA & ACA & ICA & Posterior Circulation & Unknown/Unrecorded \\
\hline No. & 1448 & 354 & 496 & 402 & 130 & 66 \\
\hline Age (yrs) & $61.9 \pm 13.4$ & $61.6 \pm 12.7$ & $60.8 \pm 13.4$ & $62.6 \pm 14.2$ & $64.1 \pm 13.2$ & $62.8 \pm 12.2$ \\
\hline Female sex & $1010(70)$ & $248(70)$ & $288(58)$ & $322(80)$ & $113(87)$ & $39(59)$ \\
\hline Acute hydrocephalus & $593(41.0)$ & $120(33.9)$ & $218(44.0)$ & $152(37.8)$ & $79(60.8)$ & $24(36.4)$ \\
\hline Clipping alone & $1073(74.1)$ & $320(90.4)$ & $385(77.6)$ & $296(73.6)$ & $51(39.2)$ & $21(31.8)$ \\
\hline Coiling alone & $285(19.7)$ & $20(5.7)$ & $97(19.6)$ & $91(22.6)$ & $70(53.9)$ & $7(10.6)$ \\
\hline Hypertension & $718(49.6)$ & $189(53.4)$ & $241(48.6)$ & $181(45.0)$ & $74(56.9)$ & $33(50.0)$ \\
\hline Diabetes mellitus & $112(7.7)$ & $20(5.7)$ & $34(6.9)$ & $34(8.5)$ & $11(8.5)$ & $13(19.7)$ \\
\hline Dyslipidemia & $180(12.4)$ & $39(11.0)$ & 65 (13.1) & $54(13.4)$ & $17(13.1)$ & $5(7.6)$ \\
\hline Smoking habit & $444(30.7)$ & $114(32.2)$ & $177(35.7)$ & $102(25.4)$ & $33(25.4)$ & $18(27.3)$ \\
\hline Alcohol consumption & $499(34.5)$ & $135(38.1)$ & $192(38.7)$ & $117(29.1)$ & $30(23.1)$ & $25(37.9)$ \\
\hline NIH stroke scale & $13.2 \pm 16.1$ & $13.29 \pm 15.7$ & $12.4 \pm 16.2$ & $12.5 \pm 16.1$ & $16.1 \pm 16.4$ & $15.1 \pm 17.9$ \\
\hline \multicolumn{7}{|l|}{ WFNS scale } \\
\hline Grade I & $484(33.4)$ & $116(32.8)$ & 169 (34.1) & $145(36.1)$ & $27(20.8)$ & $27(40.9)$ \\
\hline Grade II & $360(24.9)$ & $71(20.1)$ & $134(27.0)$ & $100(24.9)$ & $41(31.5)$ & $14(21.2)$ \\
\hline Grade III & $124(8.6)$ & $35(9.9)$ & $45(9.1)$ & $32(8.0)$ & $9(6.9)$ & $3(4.6)$ \\
\hline Grade IV & $282(19.5)$ & $86(24.3)$ & $79(15.9)$ & $76(18.9)$ & $33(25.4)$ & $8(12.1)$ \\
\hline Grade V & $194(13.4)$ & $44(12.4)$ & $69(13.9)$ & $47(11.7)$ & $20(15.4)$ & $14(21.2)$ \\
\hline \multicolumn{7}{|l|}{ Hunt \& Kosnik scale } \\
\hline Grade I & $160(11.0)$ & $36(10.2)$ & $61(12.3)$ & $40(10.0)$ & $9(6.9)$ & $14(21.2)$ \\
\hline Grade II & $568(39.2)$ & $132(37.3)$ & 198 (39.9) & $168(41.8)$ & $46(35.4)$ & $24(36.4)$ \\
\hline Grade III & $326(22.5)$ & 76 (21.5) & $110(22.2)$ & $93(23.1)$ & $38(29.2)$ & $9(13.6)$ \\
\hline Grade IV & $248(17.1)$ & $78(22.0)$ & 76 (15.3) & 69 (17.2) & $19(14.6)$ & $6(9.1)$ \\
\hline Grade V & $144(9.9)$ & $32(9.0)$ & $51(10.3)$ & $30(7.5)$ & $18(13.8)$ & $13(19.7)$ \\
\hline \multicolumn{7}{|l|}{ Fisher CT scale } \\
\hline Grade 1 & $41(2.8)$ & $7(2.0)$ & $10(2.0)$ & $14(3.5)$ & $5(3.9)$ & $5(7.6)$ \\
\hline Grade 2 & $220(15.2)$ & $49(13.8)$ & $66(13.3)$ & 77 (19.2) & $16(12.3)$ & $12(18.2)$ \\
\hline Grade 3 & $957(66.1)$ & $217(61.3)$ & $345(69.6)$ & $263(65.4)$ & 95 (73.1) & $37(56.1)$ \\
\hline Grade 4 & $227(15.7)$ & $81(22.9)$ & $73(14.7)$ & $47(11.7)$ & $14(10.8)$ & $12(18.2)$ \\
\hline
\end{tabular}

* Values are mean \pm SD or number (\%).

$\dagger$ Data for some patients were not recorded: treatment methods (clipping or coiling), 9 patients ( 3 with MCA aneurysm, 3 with ACA aneurysm, 2 with ICA aneurysm, 1 with no aneurysm location information); history of hypertension, 41 patients ( 9 with MCA aneurysm, 16 with ACA aneurysm, 13 with ICA aneurysm, 3 with no aneurysm location information); history of diabetes mellitus, 29 patients (11 with MCA aneurysm, 6 with ACA aneurysm, 11 with ICA aneurysm, 1 with posterior circulation aneurysm); history of dyslipidemia, 108 patients (24 with MCA aneurysm, 34 with ACA aneurysm, 38 with ICA aneurysm, 8 with posterior circulation aneurysm, 4 with no aneurysm location information); smoking habits, 249 patients ( 66 with MCA aneurysm, 90 with ACA aneurysm, 63 with ICA aneurysm, 22 with posterior circulation aneurysm, 8 with no aneurysm location information); alcohol consumption, 248 patients ( 64 with MCA aneurysm, 91 with ACA aneurysm, 62 with ICA aneurysm, 24 with posterior circulation aneurysm, 7 with no aneurysm location information); WFNS scale, 4 patients (2 with MCA aneurysm, 2 with ICA aneurysm); Hunt \& Kosnik scale, 2 patients (with ICA aneurysm); and Fisher CT scale, 3 patients ( 2 with ACA aneurysm, 1 with ICA aneurysm).

SAH caused by ACA aneurysm rupture tended to have lower grades on the WFNS and Hunt and Kosnik scales and was seen in younger males, and $78 \%$ of the aneurysms were treated with clipping alone. SAH caused by posterior circulation aneurysms occurred more often in older females, had a higher concurrence of acute hydrocephalus, had higher grades on the WFNS, Hunt and Kosnik, and Fisher scales, and was treated more often with endovascular coiling rather than with microsurgical clipping. Table 2 lists the age-adjusted and multivariate odds ratios for the development of sNPH. Significant independent predictors for the development of sNPH were age of 60 years or older, concurrence of acute hydrocephalus, and high grades on the WFNS, Hunt and Kosnik, and Fisher scales.
Patients with a ruptured ACA aneurysm had twice the risk for sNPH than those with a ruptured MCA aneurysm; ruptured posterior circulation aneurysms did not confer any significant risk for sNPH (Table 2). The sNPH risk in patients with an ACA aneurysm did not change even after stratification for the coexistence of acute hydrocephalus (data not shown). For patients with Fisher CT Group 1 or $2 \mathrm{SAH}$, which indicates a small hematoma volume, the ACA and ICA aneurysms conferred a 5.5- and 2.5-timeshigher risk for sNPH than MCA aneurysms, respectively (Table 3). In the subgroup of patients with Fisher Group $3 \mathrm{SAH}$, the sNPH risk conferred by ACA aneurysms was 1.8 times higher than that by the MCA aneurysms. In the subgroup of patients with Fisher Group 4 SAH, however, 
TABLE 2. Risk of coexisting sNPH after aneurysmal SAH

\begin{tabular}{|c|c|c|c|c|c|c|}
\hline Variable & sNPH & No sNPH & $\mathrm{aOR}^{*}(95 \% \mathrm{Cl})$ & $\mathrm{p}$ Value $\dagger$ & mOR $\ddagger(95 \% \mathrm{Cl})$ & p Value \\
\hline Age ( $\geq 60$ yrs) & 366 & 456 & $2.44(1.94-3.10)$ & $<0.001$ & $1.96(1.47-2.62)$ & $<0.001$ \\
\hline Acute hydrocephalus & 321 & 272 & $3.62(2.87-4.57)$ & $<0.001$ & $3.60(2.77-4.69)$ & $<0.001$ \\
\hline \multicolumn{7}{|l|}{ Aneurysm location } \\
\hline MCA & 104 & 250 & Reference & \multicolumn{3}{|c|}{ Reference } \\
\hline ACA & 211 & 285 & $1.93(1.42-2.61)$ & $<0.001$ & $2.02(1.39-2.92)$ & $<0.001$ \\
\hline ICA & 143 & 259 & $1.12(0.96-1.32)$ & 0.160 & $1.14(0.94-1.39)$ & 0.180 \\
\hline Posterior circulation & 47 & 83 & $1.06(0.91-1.23)$ & 0.450 & $1.06(0.88-1.28)$ & 0.530 \\
\hline \multicolumn{7}{|l|}{ Treatment } \\
\hline Coiling alone & 95 & 190 & Reference & \multicolumn{3}{|c|}{ Reference } \\
\hline Clipping alone & 395 & 678 & $1.59(1.18-2.14)$ & 0.002 & $1.97(1.36-2.85)$ & $<0.001$ \\
\hline \multicolumn{7}{|l|}{ Hunt \& Kosnik scale } \\
\hline Grade I or II & 159 & 569 & Reference & \multicolumn{3}{|c|}{ Reference } \\
\hline Grade III & 150 & 176 & $2.92(2.17-3.91)$ & $<0.001$ & $2.32(1.63-3.29)$ & $<0.001$ \\
\hline Grade IV or V & 212 & 180 & $3.78(2.88-4.96)$ & $<0.001$ & $2.62(1.85-3.73)$ & $<0.001$ \\
\hline \multicolumn{7}{|l|}{ Fisher CT rating scale } \\
\hline Grade 1 or 2 & 52 & 209 & Reference & \multicolumn{3}{|c|}{ Reference } \\
\hline Grade 3 & 362 & 595 & $2.15(1.53-3.01)$ & $<0.001$ & $1.95(1.30-2.91)$ & 0.001 \\
\hline Grade 4 & 106 & 121 & $3.34(2.21-5.05)$ & $<0.001$ & $2.99(1.75-5.10)$ & $<0.001$ \\
\hline
\end{tabular}

there was no significant difference among the 4 aneurysm locations. ACA aneurysms in patients with Fisher Group 3 SAH conferred a 9-fold-higher risk for sNPH than MCA aneurysms in patients with Fisher Group 1 or 2 SAH.

Among these 1448 patients, 1073 were treated with craniotomy and clipping alone, 285 were treated with coil embolization alone, and 17 underwent a combination of coiling and clipping. Microsurgical clipping conferred a 2-fold-increased risk for sNPH over coil embolization alone (Table 2). This advantage of coil embolization for the reduction of sNPH risk did not change in the subgroup of patients with Fisher Grade 3 SAH (Table 3). In the subgroup of patients with Fisher Grade 1,2, or 4 SAH, however, there was no statistically significant difference between clipping and coiling in terms of sNPH risk.

\section{Discussion}

Our large registration study revealed that ruptured ACA aneurysms conferred a significantly higher risk of sNPH than ruptured MCA aneurysms. Gruber et al. ${ }^{7}$ reported that ruptured aneurysms located in the anterior communicating artery conferred a significant increased risk for the development of sNPH compared with those in other locations $(\mathrm{p}<0.001)$. Other previous studies concluded that patients with a ruptured posterior circulation aneurysm most frequently developed sNPH; ;,5,6,12,16,17,21 3 of these 7 studies reported that patients with an anterior communicating artery or ACA aneurysm are the second-most frequent to develop sNPH and that developing sNPH after an MCA aneurysm is rare. ${ }^{6,17,21}$ The other 4 studies did not further categorize the anterior circulation aneurysms as being located in the ACA, MCA, or ICA.,5,12,16 The
sNPH risk conferred by posterior circulation aneurysms may be affected by the other modified variables, such as intraventricular hemorrhage and acute hydrocephalus, for which the frequency was reported to be the highest in patients with a ruptured posterior circulation aneurysm.9,21 We confirmed that posterior circulation aneurysms cooccurred with acute hydrocephalus more frequently and were treated more often with endovascular coiling than aneurysms in the other locations in this study. Although the patients' characteristics were similar to those in the previous studies, posterior circulation aneurysms did not confer any significantly increased risk for the development of sNPH. SAH in the interhemispheric fissure may contribute to the pathogenesis of a higher prevalence of sNPH with ruptured ACA aneurysms, because it would become an obstacle to the CSF-drainage pathway into the nasal lymphatic system via the perineural subarachnoid space enveloping the olfactory nerve rootlets. ${ }^{13,24}$

In addition, we also confirmed that increasing age, severe initial symptoms, a large-volume diffuse subarachnoid hematoma, and concurrence of acute hydrocephalus were correlated significantly with the development of sNPH; these findings are supported by previous studies. ${ }^{3-7,11,12,16,18,23}$ On the basis of the evidence of an association between the severity of $\mathrm{SAH}$ and the prevalence of subsequent sNPH, many neurosurgeons have believed that several surgical manipulations for facilitating CSF dynamics, such as hematoma evacuation, widening the opening of the cisterns, and fenestration of the lamina terminalis, might help to reduce the subsequent occurrence of sNPH after the surgical treatment of SAH. However, the authors of a recent meta-analysis of 11 nonrandomized studies in which data from 1973 patients were pooled concluded that 
TABLE 3. Risk of coexisting sNPH after aneurysmal SAH in the Fisher CT rating scale subgroups

\begin{tabular}{|c|c|c|c|c|}
\hline Variable & sNPH & No sNPH & $\mathrm{mOR}^{*}(95 \% \mathrm{Cl})$ & p Value $†$ \\
\hline Fisher Grade 1 or 2 & 52 & 209 & & \\
\hline Age ( $\geq 60$ yrs) & 36 & 93 & $2.28(0.96-5.4)$ & 0.061 \\
\hline Acute hydrocephalus & 35 & 34 & $11.1(4.64-26.5)$ & $<0.001$ \\
\hline MCA aneurysm & 4 & 52 & Reference & \\
\hline ACA aneurysm & 16 & 60 & $5.50(1.20-25.4)$ & 0.028 \\
\hline ICA aneurysm & 22 & 69 & $2.50(1.10-5.70)$ & 0.029 \\
\hline Posterior circulation aneurysm & 5 & 16 & $0.92(0.39-2.20)$ & 0.848 \\
\hline Coiling alone & 8 & 39 & Reference & \\
\hline Clipping alone & 36 & 158 & $1.67(0.47-6.00)$ & 0.430 \\
\hline Hunt \& Kosnik scale Grade I or II & 33 & 189 & Reference & \\
\hline Hunt \& Kosnik scale Grade III & 9 & 15 & $1.70(0.46-6.28)$ & 0.420 \\
\hline Hunt \& Kosnik scale Grade IV or V & 10 & 4 & $7.00(6.10-790)$ & $<0.001$ \\
\hline Fisher Grade 3 & 362 & 595 & & \\
\hline Age ( $\geq 60$ yrs) & 259 & 301 & $1.94(1.38-2.74)$ & $<0.001$ \\
\hline Acute hydrocephalus & 217 & 195 & $3.04(2.24-4.14)$ & $<0.001$ \\
\hline MCA aneurysm & 70 & 147 & Reference & \\
\hline ACA aneurysm & 154 & 191 & $1.76(1.15-2.70)$ & 0.009 \\
\hline ICA aneurysm & 92 & 171 & $1.03(0.82-1.29)$ & 0.820 \\
\hline Posterior circulation aneurysm & 36 & 59 & $1.05(0.85-1.29)$ & 0.670 \\
\hline Coiling alone & 76 & 140 & Reference & \\
\hline Clipping alone & 257 & 419 & $1.90(1.28-2.83)$ & 0.002 \\
\hline Hunt \& Kosnik scale Grade I or II & 107 & 343 & Reference & \\
\hline Hunt \& Kosnik scale Grade III & 129 & 138 & $2.41(1.64-3.56)$ & $<0.001$ \\
\hline Hunt \& Kosnik scale Grade IV or V & 126 & 114 & $2.42(1.61-3.64)$ & $<0.001$ \\
\hline Fisher Grade 4 & 106 & 121 & & \\
\hline Age ( $\geq 60$ yrs) & 70 & 61 & $2.40(1.12-5.05)$ & 0.024 \\
\hline Acute hydrocephalus & 68 & 43 & $3.29(1.63-6.66)$ & $<0.001$ \\
\hline MCA aneurysm & 30 & 51 & Reference & \\
\hline ACA aneurysm & 40 & 33 & $2.28(0.88-5.90)$ & 0.090 \\
\hline ICA aneurysm & 29 & 18 & $1.49(0.88-2.52)$ & 0.140 \\
\hline Posterior circulation aneurysm & 6 & 8 & $0.93(0.53-1.60)$ & 0.786 \\
\hline Coiling alone & 11 & 11 & Reference & \\
\hline Clipping alone & 91 & 93 & $5.00(0.86-29.2)$ & 0.073 \\
\hline Hunt \& Kosnik scale Grade I or II & 18 & 36 & Reference & \\
\hline Hunt \& Kosnik scale Grade III & 12 & 23 & $2.09(0.54-8.02)$ & 0.284 \\
\hline Hunt \& Kosnik scale Grade IV or V & 76 & 62 & $2.56(1.10-5.96)$ & 0.029 \\
\hline
\end{tabular}

* $\mathrm{mOR}$ indicates the multivariate OR for sNPH after adjustment for age, sex, hypertension, smoking habit, acute hydrocephalus, and Fisher CT rating scale grade.

† Boldface indicates statistically significant variables.

there was no significant difference in the prevalence of sNPH between the 975 patients who had undergone fenestration of the lamina terminalis and the 998 who had not ( $p$ $=0.09) \cdot{ }^{11}$ On the basis of this evidence, guidelines for the management of chronic hydrocephalus after aneurysmal SAH from the American Heart Association recommend that fenestration of the lamina terminalis not be routinely performed..$^{2}$ Another meta-analysis of 5 nonrandomized studies with data from 1718 patients pooled ${ }^{3}$ reported that 1336 patients treated with microsurgical clipping had a significantly lower risk of sNPH than 382 patients treated with endovascular coiling (relative risk $0.74 ; 95 \% \mathrm{CI}$
$0.58-0.94 ; \mathrm{p}=0.01$ ); however, 3 of the 5 studies found no significant difference between clipping and coiling for the predictive risk of $\mathrm{SNPH}^{8,15,19}$ Our results provide the first evidence that, compared with microsurgical clipping alone, coil embolization alone significantly decreased the risk for the development of sNPH. This finding may support the view that microsurgical manipulation accelerates inflammation of CSF in the subarachnoid space and leads to stagnating CSF that results from thickness and fibrosis of the arachnoid and pia mater.

The advantage of this study includes a much larger sample size than those of previous studies. Despite this 
advantage, a register-based study also has some limitations, such as recording bias. In fact, one-third of the 4693 patients registered as having had aneurysmal SAH were included in this study, and $95 \%$ of them were treated with coiling or clipping.

\section{Conclusions}

Our findings indicate that sNPH after SAH frequently occurs among patients with high-grade SAH caused by a ruptured ACA aneurysm and patients treated with microsurgical clipping. On the contrary, patients with low-grade SAH caused by a ruptured MCA aneurysm and patients treated with endovascular coiling might be less likely to develop sNPH. Studies are needed to elucidate the mechanisms by which ruptured ACA aneurysms and microsurgical treatment can increase the risk for developing sNPH after SAH.

\section{Appendix: Japan Standard Stroke Registry Study Group}

The institutions that presently contribute to the registration of hospital records for cerebral strokes are as follows:

1. Teine Keijinnkai Hospital, Department of Neurosurgery

2. Hakodate Neurosurgical Hospital, Department of Neurosurgery

3. Hokkaido Neurosurgical Memorial Hospital, Department of Neurosurgery

4. Nakamura Memorial Hospital, Stroke Center

5. Hokkaido University Hospital, Department of Neurosurgery

6. Keiwakai Ebetsu Hospital, Department of Neurosurgery

7. Asahikawa Medical University Hospital, Department of Neurology

8. Rumoi Central Clinic, Department of Neurosurgery

9. Iwate Medical University, Department of Neurology and Neurosurgery

10. Iwate Prefectural Kuji Hospital, Department of Neurosurgery

11. Southern Tohoku Research Institute for Neuroscience Hospital, Stroke Center

12. Kohnan Hospital, Stroke Center

13. Research Institute for Brain and Blood Vessels Akita, Stroke Center

14. Saiseikai Central Hospital, Department of Neurology

15. Kanto Medical Center NTT East Corporation, Department of Neurosurgery

16. Keio University School of Medicine, Department of Neurology

17. National Center for Global Health and Medicine, Neurosurgery

18. Hatanodai Neurosurgical Hospital, Department of Neurosurgery

19. Metroporitan Ohkubo Hospital, Department of Neurosurgery

20. Tokyo Women's Medical University Hospital, Department of Neurology

21. Kyorin University Hospital, Department of Neurosurgery

22. Akiru Municipal Medical Center, Department of Neurosurgery

23. Tokyo Medical University Hachioji Medical Center, Department of Neurosurgery

24. Yokohama Rosai Hospital, Department of Neurology

25. Kawasaki Saiwai Hospital, Department of Neurosurgery

26. Sagamihara Kyoudo Hospital, Department of Neurosurgery

27. Yokohama Stroke \& Brain Center

28. Yokohama City University Hospital, Stroke Center

29. Shonankamakura General Hospital, Stroke Center

30. Tokai University, Department of Oiso Hospital, Department of Neurology

31. Tokai University Hospital, Department of Neurology
32. Tokyo Dental College Ichikawa General Hospital, Department of Neurosurgery

33. Jisenkai Yoshida Hospital, Department of Neurosurgery

34. Chiba Rosai Hospital, Department of Neurosurgery

35. Chiba Cardiovascular Center, Stroke Center

36. Dokkyo University School of Medicine, Department of Neurology

37. Ryugasaki Saiseikai Hospital, Department of Neurology

38. Saitama International Medical Research Center Hospital,

Stroke Center

39. Hiratsuka Kyosai Hospital, Stroke Center

40. Saitama Medical University Hospital, Department of Neurology

41. Fukui University Hospital, Department of Neurology

42. Toyama University Hospital, Department of Neurology and Neurosurgery

43. Shizuoka General Hospital, Department of Neurology

44. Matsunami General Hospital, Department of Neurosurgery

45. Shiga University of Medical Science Hospital, Department of Neurology and Neurosurgery

46. Osaka University Faculty of Medicine, Department of Neurology

47. National Cardiovascular Center, Department of Cerebrovascular Disease

48. Hoshigaoka Koseinenkin Hospital, Department of Neurology

49. Kyoto Second Red Cross Hospital, Department of Neurology 50. Kyoto Prefectural University of Medicine, Department of Neurology

51. Otowa Hospital, Department of Neurology

52. Itami City Hospital, Department of Neurosurgery

53. Kobe City General Hospital, Stroke Center

54. Hyogo Brain and Heart Center, Department of Neurology

55. Matsue City Hospital, Department of Neurosurgery

56. Shimane Prefectural Central Hospital, Department of Neurology and Neurosurgery

57. Shimane University Hospital, Department of Neurology and Neurosurgery

58. Okayama Rosai Hospital, Department of Neurosurgery

59. Matsue Red Cross Hospital, Department of Neurology

60. Ohda Municipal Hospital, Department of Neurology

61. Masuda Red Cross Hospital, Department of Neurology

62. Kawasaki Medical School Hospital, Stroke Center

63. National Okayama Medical Center, Department of Neurology

64. Okayama Kyokuto Hospital, Department of Cerebrovascular

Disease

65. Konan Central Hill Hospital, Department of Neurosurgery

66. Oota Memorial Hospital, Department of Neurology

67. Okayama University Hospital, Department of Neurology

68. National Hospital Organization Okayama Medical Center,

Department of Neurology

69. Kajikawa Hospital, Department of Neurology

70. Hiroshima University Hospital, Department of Neurology

71. National Hospital Organization Kure Medical Center, Department of Neurology

72. Higashihiroshima Medical Center, Department of Neurology

73. Yamaguchi University Hospital, Department of Neurosurgery

74. Kagawa University Hospital, Department of Neurology

75. Osaka Neurosurgical Hospital, Department of Rehabilitation

76. Tokushima University Hospital, Department of Neurosurgery

77. Ehime University Hospital, Department of Neurosurgery

78. Chikamori Hospital, Department of Neurology

79. Saiseikai Fukuoka General Hospital, Department of Neurology

80. Kyusyu University Hospital, Stroke Center

81. Kyusyu National Medical Center Hospital, Department of Neurology

82. Iizuka Hospital, Department of Neurology 
83. St. Mary's Hospital, Cerebral Stroke Center

84. Saga Prefectural Hospital, Department of Neurosurgery 85. Saiseikai Kumamoto Hospital, Department of Neurology 86. Kumamoto City Hospital, Department of Neurology 87. Kumamoto Red Cross Hospital, Department of Neurology 88. Fukuoka Red Cross Hospital, Department of Neurology 89. Oita Almedia Hospital, Department of Neurosurgery 90. Miyakonojo Regional Medical Center, Department of Neurosurgery

91. Ryukyu University Hospital, Department of Neurology

92. Okinawa Prefectural Yaeyama Hospital, Department of Neurosurgery

93. Fukuoka Tokusyukai Hospital, Department of Neurology

94. Kohase Hospital, Department of Neurosurgery

\section{References}

1. Chan M, Alaraj A, Calderon M, Herrera SR, Gao W, Ruland $S$, et al: Prediction of ventriculoperitoneal shunt dependency in patients with aneurysmal subarachnoid hemorrhage. J Neurosurg 110:44-49, 2009

2. Connolly ES Jr, Rabinstein AA, Carhuapoma JR, Derdeyn CP, Dion J, Higashida RT, et al: Guidelines for the management of aneurysmal subarachnoid hemorrhage: a guideline for healthcare professionals from the American Heart Association/American Stroke Association. Stroke 43:1711-1737, 2012

3. de Oliveira JG, Beck J, Setzer M, Gerlach R, Vatter H, Seifert $\mathrm{V}$, et al: Risk of shunt-dependent hydrocephalus after occlusion of ruptured intracranial aneurysms by surgical clipping or endovascular coiling: a single-institution series and metaanalysis. Neurosurgery 61:924-934, 2007

4. Dehdashti AR, Rilliet B, Rufenacht DA, de Tribolet N: Shunt-dependent hydrocephalus after rupture of intracranial aneurysms: a prospective study of the influence of treatment modality. J Neurosurg 101:402-407, 2004

5. Dorai Z, Hynan LS, Kopitnik TA, Samson D: Factors related to hydrocephalus after aneurysmal subarachnoid hemorrhage. Neurosurgery 52:763-771, 2003

6. Graff-Radford NR, Torner J, Adams HP Jr, Kassell NF: Factors associated with hydrocephalus after subarachnoid hemorrhage. A report of the Cooperative Aneurysm Study. Arch Neurol 46:744-752, 1989

7. Gruber A, Reinprecht A, Bavinzski G, Czech T, Richling B: Chronic shunt-dependent hydrocephalus after early surgical and early endovascular treatment of ruptured intracranial aneurysms. Neurosurgery 44:503-512, 1999

8. Jartti P, Karttunen A, Isokangas JM, Jartti A, Koskelainen T, Tervonen O: Chronic hydrocephalus after neurosurgical and endovascular treatment of ruptured intracranial aneurysms. Acta Radiol 49:680-686, 2008

9. Kallmes DF, Lanzino G, Dix JE, Dion JE, Do H, Woodcock RJ, et al: Patterns of hemorrhage with ruptured posterior inferior cerebellar artery aneurysms: CT findings in 44 cases. AJR Am J Roentgenol 169:1169-1171, 1997

10. Kobayashi S: International experience in stroke registry: Japanese Stroke DataBank. Am J Prev Med 31 (6 Suppl 2):S240-S242, 2006

11. Komotar RJ, Hahn DK, Kim GH, Starke RM, Garrett MC, Merkow MB, et al: Efficacy of lamina terminalis fenestration in reducing shunt-dependent hydrocephalus following aneurysmal subarachnoid hemorrhage: a systematic review. Clinical article. J Neurosurg 111:147-154, 2009

12. Little AS, Zabramski JM, Peterson M, Goslar PW, Wait SD, Albuquerque FC, et al: Ventriculoperitoneal shunting after aneurysmal subarachnoid hemorrhage: analysis of the indications, complications, and outcome with a focus on patients with borderline ventriculomegaly. Neurosurgery 62:618627, 2008

13. McComb JG: Recent research into the nature of cerebrospinal fluid formation and absorption. J Neurosurg 59:369-383, 1983
14. Mehta V, Holness RO, Connolly K, Walling S, Hall R: Acute hydrocephalus following aneurysmal subarachnoid hemorrhage. Can J Neurol Sci 23:40-45, 1996

15. Mura J, Rojas-Zalazar D, Ruíz A, Vintimilla LC, Marengo JJ: Improved outcome in high-grade aneurysmal subarachnoid hemorrhage by enhancement of endogenous clearance of cisternal blood clots: a prospective study that demonstrates the role of lamina terminalis fenestration combined with modern microsurgical cisternal blood evacuation. Minim Invasive Neurosurg 50:355-362, 2007

16. O'Kelly CJ, Kulkarni AV, Austin PC, Urbach D, Wallace MC: Shunt-dependent hydrocephalus after aneurysmal subarachnoid hemorrhage: incidence, predictors, and revision rates. Clinical article. J Neurosurg 111:1029-1035, 2009

17. Pietilä TA, Heimberger KC, Palleske H, Brock M: Influence of aneurysm location on the development of chronic hydrocephalus following SAH. Acta Neurochir (Wien) 137:7073, 1995

18. Rincon F, Gordon E, Starke RM, Buitrago MM, Fernandez A, Schmidt JM, et al: Predictors of long-term shunt-dependent hydrocephalus after aneurysmal subarachnoid hemorrhage. Clinical article. J Neurosurg 113:774-780, 2010

19. Sethi H, Moore A, Dervin J, Clifton A, MacSweeney JE: Hydrocephalus: comparison of clipping and embolization in aneurysm treatment. J Neurosurg 92:991-994, 2000

20. Takizawa S, Shibata T, Takagi S, Kobayashi S: Seasonal variation of stroke incidence in Japan for 35631 stroke patients in the Japanese Standard Stroke Registry, 1998-2007. J Stroke Cerebrovasc Dis 22:36-41, 2013

21. Tapaninaho A, Hernesniemi J, Vapalahti M, Niskanen M, Kari A, Luukkonen M, et al: Shunt-dependent hydrocephalus after subarachnoid haemorrhage and aneurysm surgery: timing of surgery is not a risk factor. Acta Neurochir (Wien) 123:118-124, 1993

22. Varelas P, Helms A, Sinson G, Spanaki M, Hacein-Bey L: Clipping or coiling of ruptured cerebral aneurysms and shunt-dependent hydrocephalus. Neurocrit Care 4:223-228, 2006

23. Yang TC, Chang CH, Liu YT, Chen YL, Tu PH, Chen HC: Predictors of shunt-dependent chronic hydrocephalus after aneurysmal subarachnoid haemorrhage. Eur Neurol 69:296303, 2013

24. Weller RO, Kida S, Zhang ET: Pathways of fluid drainage from the brain-morphological aspects and immunological significance in rat and man. Brain Pathol 2:277-284, 1992

\section{Author Contributions}

Conception and design: Yamada, Ishikawa, Kobayashi. Acquisition of data: Kobayashi. Analysis and interpretation of data: Yamada, Ishikawa, Yamamoto, Kobayashi. Drafting the article: Ishikawa, Yamamoto, Kobayashi. Approved the final version of the manuscript on behalf of all authors: Yamada. Statistical analysis: Yamada, Ishikawa, Kobayashi. Administrative/technical/material support: Ishikawa, Ino, Kimura, Kobayashi. Study supervision: Ishikawa, Yamamoto, Ino, Kimura, Kobayashi. Organized the Japanese Stroke DataBank: Kobayashi.

\section{Supplemental Information Current Affiliation \\ Dr. Kimura: Rakuwakai Otowa Rehabilitation Hospital, Kyoto, Japan.}

\section{Correspondence}

Shigeki Yamada, Department of Neurosurgery and Stroke Center, Normal Pressure Hydrocephalus Center, Rakuwakai Otowa Hospital, Otowachinji-cho 2, Yamashina-ku, Kyoto 607-8602, Japan. email: shigekiyamada3@gmail.com. 